\title{
Interplay of bulk and surface properties for steady-state measurements of minority carrier lifetimes
}

Marko Turek 1, a)

Fraunhofer-Center for Silicon Photovoltaics CSP

(Dated: 29 October 2018)

The measurement of the minority carrier lifetime is a powerful tool in the field of semiconductor material characterization as it is very sensitive to electrically active defects. Furthermore, it is applicable to a wide range of samples such as ingots or wafers. In this work, a systematic theoretical analysis of the steady-state approach is presented. It is shown how the measured lifetime relates to the intrinsic bulk lifetime for a given material quality, sample thickness, and surface passivation. This makes the bulk properties experimentally accessible by separating them from the surface effects. In particular, closed analytical solutions of the most important cases, such as passivated and unpassivated wafers and blocks are given. Based on these results, a criterion for a critical sample thickness is given beyond which a lifetime measurement allows deducing the bulk properties for a given surface recombination. These results are of particular interest for semiconductor material diagnostics especially for photovoltaic applications but not limited to this field.

\footnotetext{
a) Electronic mail: marko.turek@csp.fraunhofer.de
} 


\section{INTRODUCTION}

Minority carrier lifetime measurements have been proven to be an essential material characterization technique for semiconductors. The determination of the lifetime provides information on electrically active defects in a very sensitive way. It is possible to detect defect concentrations down to $10^{10} \mathrm{~cm}^{-3}$ with contactless methods at room temperature. $\underline{1}, \underline{2}$

There are different approaches which are based on either a dynamic process taking place after a pulsed carrier generation (transient measurement) or a steady-state measurement during a continuous carrier generation. In either case, the measured lifetime $\tau_{\text {eff }}$ incorporates both bulk and surface recombination processes. However, a typical requirement on the lifetime measurement is that either the material properties, i.e. bulk lifetime $\tau_{\mathrm{b}}$, or the surface properties, i.e. surface recombination velocity $S$, can be unambiguously deduced. The first information on the bulk allows, for example, an optimization of the production process of the semiconductor, i.e. the crystallization. On the other hand, the second information on the surface recombination plays an important role, for example, for a device optimization like the development of the passivation layers for solar cells. Therefore, it is inevitable to know the precise relationship between the measured lifetime $\tau_{\text {eff }}$, the bulk lifetime $\tau_{\mathrm{b}}$ and the surface recombination velocity $S$, i.e. the function $\tau_{\text {eff }}=\tau_{\text {eff }}\left(\tau_{\mathrm{b}}, S\right)$. Typically, this relationship depends on sample properties such as its thickness $d$ as well as the spectrum of the light generating the carriers and the corresponding absorption length $\alpha^{-1}$.

For transient measurements and equal surface recombination velocities on the front and back side of the sample $\left(S_{0}=S_{1}=S\right)$, the most frequently used functional relation is given by $\tau_{\text {eff }}\left(\tau_{\mathrm{b}}, S, d\right)=\left(\tau_{\mathrm{b}}^{-1}+\tau_{\mathrm{s}, \mathrm{tr}}^{-1}\right)^{-1}$. Here, the so-called surface recombination lifetime $\tau_{\mathrm{s}, \mathrm{tr}}$ is given by the phenomenological approximation $\tau_{\mathrm{s}, \mathrm{tr}} \approx d /(2 S)+d^{2} /\left(\pi^{2} D\right)$ while $D$ stands for the diffusion constant of the minority carriers. This approximation is a superposition of two asymptotic solutions of the partial differential equation for the excess carrier density $p$ corresponding to a transient measurement. The first term is exact in the limit of very low surface recombination and thin samples while the second term is found for very high surface recombination and thick samples. $\stackrel{3.4}{*}$ To obtain this result, only the fastest decaying mode of the density after a short illumination pulse is considered which leads to a transcendental equation for $\tau_{\mathrm{s}, \mathrm{tr}}$ that can be solved in the $S d / D \ll 1$ and $S d / D \gg 1$ limits, respectively. In subsequent works, this approach has been extended to the case of unequal $S_{0} \neq S_{1}$ and a 
systematic approach based on the relevant dimensionless parameters has been presented. $\underline{\underline{5}}^{-\underline{\underline{7}}}$

The second widely-used approach relies on a steady-state measurement. A mathematical model for this case can be formulated by an ordinary differential equation, i.e. a time independent diffusion equation for the excess carrier density $p$. Despite a number of assumptions that have to be made in order to validate this model, the solution to this equation can give rather accurate results for the carrier density. $\underline{\underline{8} .9}$ However, it has been pointed out that the transient approach and the steady-state approach can lead to different results for $\tau_{\text {eff }}$ in certain cases. ${ }^{10,11}$ Furthermore, a number of experimental techniques are based on a steady-state of the sample created by a long illumination, such as lifetime measurements using photoluminescence (PL) or the determination of the (quasi) steady-state photoconductance (QSSPC) $\stackrel{12}{*}$ In addition, the dependence of $\tau_{\text {eff }}$ on the absorption length $\alpha^{-1}$ of the light used for the carrier generation as well as the influence of the surface recombination for intermediate values of $S$ is of interest.

A common approach to determine $\tau_{\mathrm{b}}$ experimentally is to prepare the samples such that the surface properties are negligible. This can be achieved by a surface passivation which significantly reduces the surface recombination velocities..$\underline{13}$ In this case, the bulk lifetime is measured directly, i.e. $\tau_{\text {eff }} \approx \tau_{\mathrm{b}}$. For small surface recombination velocities, a correction based on the limiting expression $\tau_{\mathrm{s}} \approx d /(2 S)$ can be employed to improve the accuracy of $\tau_{\mathrm{b}} \stackrel{14}{\underline{14}}$ On the other hand, a method for determining $\tau_{\mathrm{b}}$ on thick unpassivated samples, i.e. blocks, has been recently proposed. $\stackrel{15}{ }$ Furthermore, the feasibility of measuring bulk properties on thin unpassivated wafers has been investigated, both experimentally and numerically. $\underline{\underline{16}} \underline{\underline{17}}$

In this work, we will present a systematic analysis of the relation between the measured lifetime $\tau_{\text {eff }}$, the bulk property $\tau_{\mathrm{b}}$ and the surface recombination velocity $S$ that is valid for the steady-state approach. Our study is based on the function $\tau_{\text {eff }}=\tau_{\text {eff }}\left(\tau_{\mathrm{b}}, d, S_{0}, S_{1}, \alpha\right)$ that can be derived from the solution of the time independent diffusion equation, see Sec. II. It generalizes special cases obtained earlier $\underline{15} \underline{-17}$ for the steady-state regime and furthermore allows investigating all practically relevant sample types within the same framework. In particular, our study extends the previously proposed results for thin wafers on one side and thick samples such as blocks on the other side to samples of arbitrary thickness. The qualitative behaviour of this solution with respect to a variation in the parameters $\tau_{\mathrm{b}}, d$, $S_{0,1}$, and $\alpha$ will be discussed in Sec. [II]. Approximations valid for the relevant cases of homogeneous generation (i.e. $\alpha \rightarrow 0$ ), unpassivated samples (i.e. $S \rightarrow \infty$ ), and large 
sample thickness (i.e. $d \rightarrow \infty$ ) will also be given in Sec. III. These results allow for a straightforward interpretation of the data obtained in lifetime measurements. In Sec. IV a systematic approach is presented that allows extracting all relevant asymptotic limits with respect to the sample parameters $\tau_{\mathrm{b}}, S, d$, and $\alpha$. As a direct application of these asymptotic expressions a criterion for the sample thickness beyond which a reliable determination of the bulk lifetime is possible will be derived. It will be shown how this critical thickness $d_{\text {crit }}$ depends on $S, \tau_{\mathrm{b}}$ and $\alpha$. As a second application the correct expression for $\tau_{\text {eff }}$ in the limit of increasingly better material quality, i.e. $\tau_{\mathrm{b}} \rightarrow \infty$, will be deduced and compared to a phenomenological result published ealier. $\stackrel{16}{17}$ Finally, we will discuss the experimental conditions which allow the separation of bulk and surface properties in an unambiguous manner.

\section{DIFFUSION MODEL}

The measurable carrier lifetime $\tau_{\text {eff }}$ for a steady-state application is given by

$$
\tau_{\text {eff }}=\frac{N_{p}}{G} \equiv\left(\int_{-d / 2}^{d / 2} \mathrm{~d} x p(x)\right) /\left(\int_{-d / 2}^{d / 2} \mathrm{~d} x g(x)\right)
$$

with $N_{p}$ being the total number of minority excess carriers in the sample and $G$ the total generation rate in the bulk, respectively. For the steady state approach the generation rate per volume is time independent and given by

$$
g(x)=\frac{P \lambda}{A h c}(1-r) \frac{\alpha \mathrm{e}^{-\alpha d / 2}}{1-r^{2} \mathrm{e}^{-2 \alpha d}}\left(1+r \mathrm{e}^{\alpha(2 x-d)}\right) \mathrm{e}^{-\alpha x}
$$

with $P$ being the power of the illumination, $A$ the area of the sample, $\lambda$ the wavelength, $\alpha^{-1}$ the absorption length, and $r$ the reflection coefficient. $\frac{3}{\text { Th }}$ The diffusion equation and the boundary conditions, that describe the carrier density $p(x)$, read

$$
\frac{\mathrm{d}^{2} p}{\mathrm{~d} x^{2}}-\frac{p(x)}{D \tau_{\mathrm{b}}}+\frac{g(x)}{D}=0,\left.\frac{\mathrm{d} p(x)}{\mathrm{d} x}\right|_{ \pm \frac{d}{2}}=\mp \frac{S_{1,0}}{D} p\left( \pm \frac{d}{2}\right) .
$$

Integrating the diffusion equation straightforwardly yields $G=R+A\left[S_{0} p(-d / 2)+\right.$ $S_{1} p(+d / 2)$ ] with $R$ being the total recombination rate in the bulk. This allows for a simple interpretation of the surface recombination velocities: all generated carriers that are not recombining in the bulk have to recombine at the surface and these processes are characterized 
by $S_{0,1}$ and $p( \pm d / 2)$. The solution to Eq. (3) is given by

$$
p(x)=c_{1} \mathrm{e}^{x / l}+c_{2} \mathrm{e}^{-x / l}+c_{3} \mathrm{e}^{\alpha x}+c_{4} \mathrm{e}^{-\alpha x}
$$

with $l \equiv \sqrt{\tau_{\mathrm{b}} D}$ being the diffusion length.1,8,9,11,18 The prefactors $c_{1-4}$ are obtained by inserting this solution into the diffusion equation and the corresponding boundary conditions, Eq. (3). Integration of the carrier density $p(x)$ and the generation rate $g(x)$ according to Eq. (1) then yields the function $\tau_{\text {eff }}=\tau_{\text {eff }}\left(\tau_{\mathrm{b}}, S, d, \alpha\right)$.

The major assumptions for this model are:

1. lateral homogeneity of the sample allowing for a one-dimensional description;

2. constant, i.e. injection independent, material properties $\tau_{\mathrm{b}}, S$, and diffusion constant $D ;$

3. monochromatic illumination with a single wavelength $\lambda$ and corresponding absorption depth $\alpha^{-1}$

4. large penetration depth of the sensor measuring the total number of carriers $N_{p}$ in comparison to absorption length $\alpha^{-1}$ and diffusion length $l$.

The lateral homogeneity of the material has to be given on length scales larger than the diffusion length. With this condition fullfilled and given that the measurement is performed in a steady state, the variations in the material parameters are due to the position dependent carrier density $p(x)$ only. Furthermore, the model could easily be extended beyond the monochromatic case by linear superposition. However, this would make the analysis technically more complex while for the purpose of this work there are no significant additional insights to be gained.

In order to get a deeper insight into the relationship between the lifetimes, the surface properties and the sample geometry we state the result for the most relevant cases. First, in case of equal surface recombination, i.e. $S_{0}=S_{1}=S$, one finds

$$
\tau_{\mathrm{eff}}^{S_{0}=S_{1}}=\frac{\tau_{\mathrm{b}}}{1-\alpha^{2} l^{2}}\left[1-\alpha l \frac{\alpha l+\frac{S l}{D} \operatorname{coth} \frac{\alpha d}{2}}{1+\frac{S l}{D} \operatorname{coth} \frac{d}{2 l}}\right]
$$

This solution is valid for any sample thickness $d$, absorption length $\alpha^{-1}$ and surface recombination velocity $S$. Second, the result for homogenous generation but with non-equal surface recombination velocities is presented 


$$
\tau_{\mathrm{eff}}^{\alpha \rightarrow 0}=\tau_{\mathrm{b}}\left[1-\frac{\frac{\left(S_{0}+S_{1}\right) d}{D} \frac{l^{2}}{d^{2}} \sinh \left[\frac{d}{l}\right]+4 \frac{S_{0} d}{D} \frac{S_{1} d}{D} \frac{l^{3}}{d^{3}} \sinh ^{2}\left[\frac{d}{2 l}\right]}{\frac{\left(S_{0}+S_{1}\right) d}{D} \frac{l}{d} \cosh \left[\frac{d}{l}\right]+\left(1+\frac{S_{0} d}{D} \frac{S_{1} d}{D} \frac{l^{2}}{d^{2}}\right) \sinh \left[\frac{d}{l}\right]}\right] .
$$

This result applies to wafers with a thickness being small enough such that a homogeneous generation $g(x)=g$ is given, i.e. $\alpha d \ll 1$.

It is worthwhile to note, that one could in principle determine a surface recombination time $\tau_{\mathrm{s}}$ from Eq. (5) by applying $\tau_{\mathrm{s}}^{-1}=\tau_{\text {eff }}^{-1}-\tau_{\mathrm{b}}^{-1}$. However, the $\tau_{\mathrm{s}}$ obtained in this way is in general not independent of $\tau_{\mathrm{b}}$ (or, similarly, of $l=\sqrt{D \tau_{\mathrm{b}}}$ ). This is different from the case of the approximate solution obtained in the transient approach.

In a typical experiment, the quantities $\tau_{\text {eff }}, \alpha$, and $d$ are known or measurable while $\tau_{\mathrm{b}}$ and $S$ are unknown. This implies that the stated relationship $\tau_{\text {eff }}=\tau_{\text {eff }}\left(\tau_{\mathrm{b}}, d, S, \alpha\right)$, i.e. Eq. (5), in general allows for the determination of the two unknown quantities if two independent measurements are performed with either $d$ or $\alpha$ being varied. For example, a well known approach to determine $S$ is to set up an experiment where wafers of the same material and surface treatment but with different thickness are measured. $\stackrel{2}{*}$ However, as will be shown in the next section, a careful choice of range for these thicknesses has to be made if both $S$ and $\tau_{\mathrm{b}}$ are to be determined within the same experiment.

\section{APPLICATION TO WAFERS AND BLOCKS}

For the following discussion of the resulting relation $\tau_{\text {eff }}=\tau_{\text {eff }}\left(\tau_{\mathrm{b}}, S, d\right)$ we focus on the case where $\left|S_{0}-S_{1}\right| /\left(S_{0}+S_{1}\right) \ll 1$ which is reflected by Eq. (5) . The rather general result (5) can easily be simplified to yield the practically relevant expression for thin wafers with homogeneous generation, i.e. $\alpha^{-1} \gg d, l$,

$$
\tau_{\mathrm{eff}}^{\alpha \rightarrow 0}=\tau_{\mathrm{b}}\left[1-\frac{2 \frac{S l}{D} \frac{l}{d}}{1+\frac{S l}{D} \operatorname{coth} \frac{d}{2 l}}\right] .
$$

Furthermore, the case of very large surface recombination is given by

$$
\tau_{\mathrm{eff}}^{S \rightarrow \infty}=\frac{\tau_{\mathrm{b}}}{1-\alpha^{2} l^{2}}\left[1-\alpha l \frac{\tanh \frac{d}{2 l}}{\tanh \frac{\alpha d}{2}}\right]
$$

which extends the result for homogeneous carrier generation $\tau_{\text {eff }}^{\alpha \rightarrow 0, S \rightarrow \infty}=\tau_{\mathrm{b}}\{1-2(l / d) \tanh [d /(2 l)]\}$ presented in Ref. 16. For thick blocks with $d \gg l, \alpha^{-1}$ one finds

$$
\tau_{\text {eff }}^{d \rightarrow \infty}=\frac{\tau_{\mathrm{b}}}{1-\alpha^{2} l^{2}}\left[1-\alpha l \frac{\alpha l+S l / D}{1+S l / D}\right]
$$




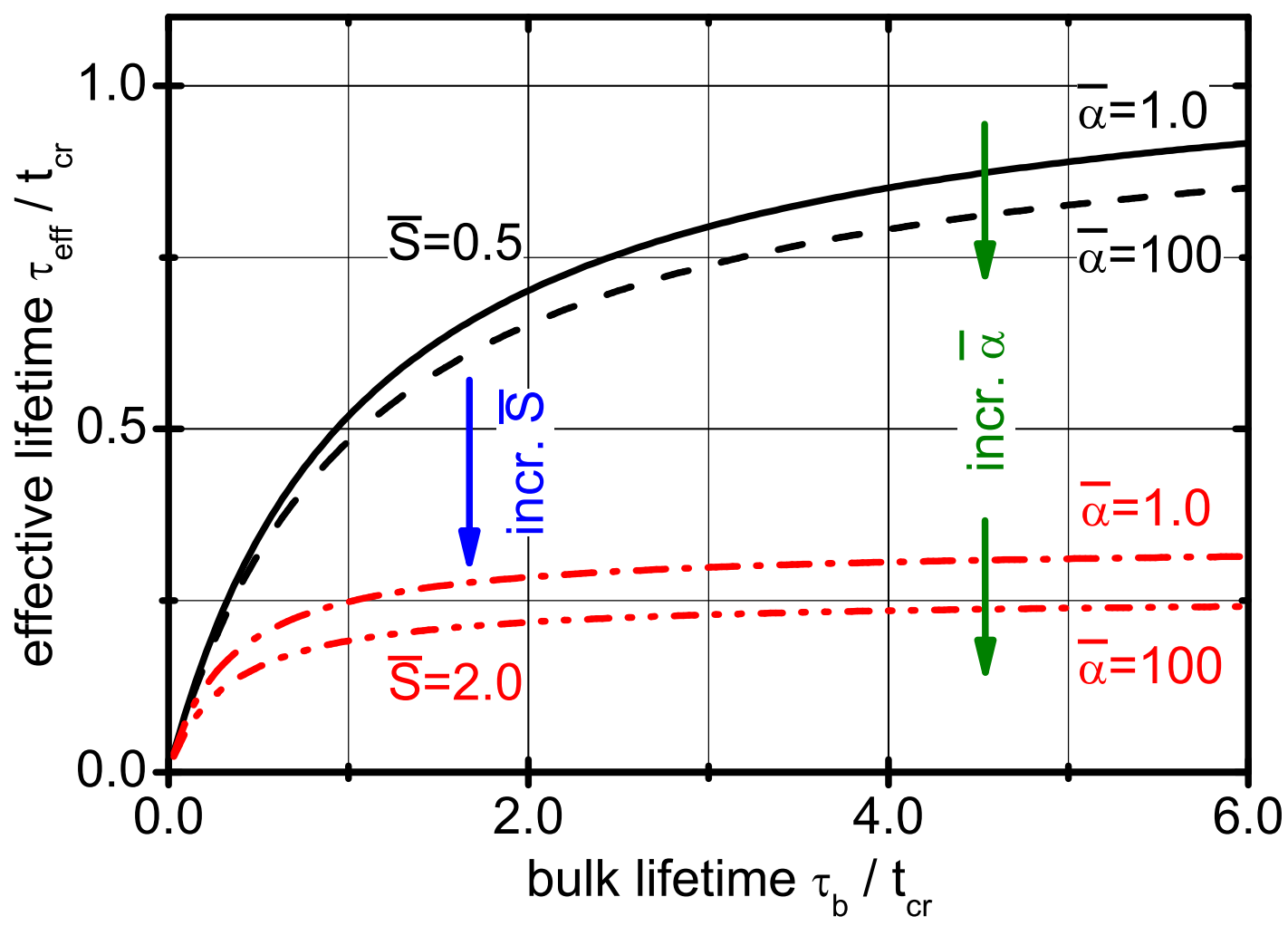

FIG. 1. (Color online) Measurable (effective) lifetime $\tau_{\text {eff }}$ (in units of $t_{\text {cr }}=d^{2} / D$ ) as a function of bulk lifetime $\tau_{\mathrm{b}}$ and for the dimensionless parameters $\bar{S}=0.5, \bar{\alpha}=1.0$ (solid, black), $\bar{S}=0.5, \bar{\alpha}=100$ (dashed, black), $\bar{S}=2.0, \bar{\alpha}=1.0$ (dash-dot, red), $\bar{S}=2.0$, $\bar{\alpha}=100$ (dash-dot-dot, red).

which is a generalization of the result given in Ref. 15 as it includes the surface recombination velocity $S$ explicitly. It turns out that the restriction $S_{0}=S_{1}$ is not necessary to obtain this result as only the front side of a block influences the total number of excess carriers significantly. Assuming the front side to be unpassivated, i.e. $S=S_{0} \rightarrow \infty$, the expression simply reproduces the result $\tau_{\text {eff }}^{d \rightarrow \infty, S \rightarrow \infty} \approx \tau_{\mathrm{b}} /(1+\alpha l)$ given in Ref. 15. Note, that the corresponding surface recombination time $\tau_{\mathrm{s}}=l /(\alpha D)$ is not covered by the phenomenological expression $\tau_{\mathrm{s}, \text { tr }}$ derived in the transient case.

Before the correct quantitative asymptotic limits are discussed in more detail, the qualitative features of relationship (5) are studied in terms of the dimensionless parameters $\bar{S} \equiv S d / D, \bar{\alpha} \equiv \alpha d$, and $\bar{l}^{2}=l^{2} / d^{2}=\tau_{\mathrm{b}} / t_{\mathrm{cr}} \equiv \bar{\tau}_{\mathrm{b}}$. Here, $t_{\mathrm{cr}} \equiv d^{2} / D$ stands for the time scale defined by the diffusion through the entire sample. The dependence of $\tau_{\text {eff }}$ on $\tau_{\mathrm{b}}$ for various parameters $\bar{S}$ and $\bar{\alpha}$ is shown in Fig. 1. One can identify a region of small bulk lifetimes 


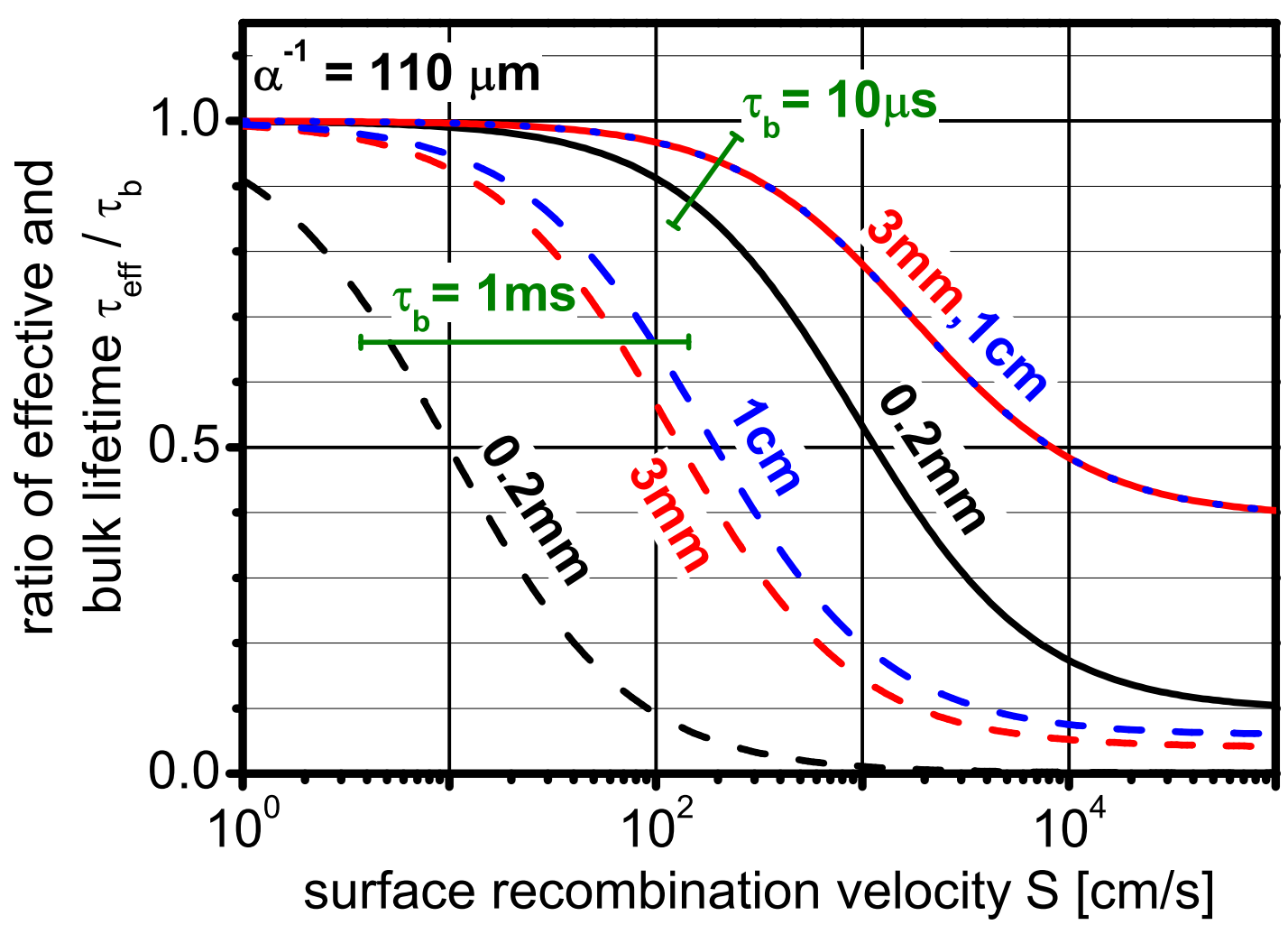

FIG. 2. (Color online) Ratio between measurable lifetime $\tau_{\text {eff }}$ and bulk lifetime $\tau_{\mathrm{b}}$ as a function of the surface recombination velocity $S$. Shown are two different material qualitites: $\tau_{\mathrm{b}}=10 \mu \mathrm{s}$ (solid lines) and $\tau_{\mathrm{b}}=1 \mathrm{~ms}$ (dashed lines). The thickness of the samples are indicated and increase from $d=200 \mu \mathrm{m}$ (left curves, black) to $d=1 \mathrm{~cm}$ (right curves, blue).

where $\tau_{\text {eff }} \approx \tau_{\mathrm{b}}$. In this region, a measurement of $\tau_{\text {eff }}$ allows a direct determination of $\tau_{\mathrm{b}}$. The size of this region increases for lower surface recombination, i.e. decreasing $\bar{S}$. On the other hand, for large bulk lifetimes the value of $\tau_{\text {eff }}$ saturates even if $\tau_{\mathrm{b}}$ is increasing further. This implies that a measurement of $\tau_{\text {eff }}$ is not sensitive to any changes in the bulk property $\tau_{\mathrm{b}}$ anymore since the surface recombination dominates. Therefore, a measurement of $\tau_{\mathrm{eff}}$ in this region can in principle not give any information about $\tau_{\mathrm{b}}$. Finally, there is a region of intermediate values for $\tau_{\mathrm{b}}$ where a direct proportionality between $\tau_{\text {eff }}$ and $\tau_{\mathrm{b}}$ is not given. However, the information gained in a measurement of $\tau_{\text {eff }}$ could still be used to determine $\tau_{\mathrm{b}}$. In the next section, these features will be discussed in a more formal and quantitative way.

The influence of the surface passivation on the measurement is presented in more de- 
tail in Fig. 2, As already noted, the measured lifetime is approximately equal to the bulk lifetime when the surface recombination is sufficiently suppressed. The importance of the surface passivation decreases with increasing sample thickness and bulk recombination. Furthermore, there is another important aspect to be seen in Fig. 2, For parameter regimes where $S$ is either small or large, the ratio $\tau_{\text {eff }} / \tau_{\mathrm{b}}$ becomes constant and thus significantly less sensitive to the precise value of $S$. It implies that the bulk value $\tau_{\mathrm{b}}$ can be concluded from $\tau_{\text {eff }}$ for large sample thickness and large surface recombination even if the precise value of $S$ is not known. This justifies the approach in Ref. 15. In the intermediate region (in Fig. 2 roughly between $S \sim 10 \mathrm{~cm} / \mathrm{s}$ and $S \sim 10^{4} \mathrm{~cm} / \mathrm{s}$ ) a detailed knowledge of the surface recombination velocity is necessary in order to determine $\tau_{\mathrm{b}}$ from a measurement.

Compared to the strong dependence of the ratio $\tau_{\text {eff }} / \tau_{\mathrm{b}}$ on $\bar{S}$, the influence of $\bar{\alpha}$ is rather weak, see Fig. 1, Nevertheless, this dependence can be employed by measuring the same sample two or more times injecting carriers with light of different wave lengths. These wavelength dependent measurements allow elliminating other unknown quantities such as $S \underline{18,19}$

The sample thickness is the third relevant parameter. In Fig. 2, one can observe that for increasing thickness a limiting curve is approached which means that an increase in $d$ beyond a certain critical thickness does not change the measurement result much. This can also be seen from $\tau_{\text {eff }}^{d \rightarrow \infty}$ which is independent of $d$. The critical thickness is lower for materials with lower bulk lifetime. The relation between $\tau_{\text {eff }}$ and $\tau_{\mathrm{b}}$ as a function of $d$ itself is presented in Fig. 3. The ratio $\tau_{\text {eff }} / \tau_{\mathrm{b}}$ reaches a constant value smaller than one for very large thickness, i.e. $d \rightarrow \infty$. This implies that although $\tau_{\text {eff }}$ is always smaller than $\tau_{\mathrm{b}}$ there is nevertheless a proportionality between these two quantities. Therefore, it is possible to deduce the bulk property from a measurement of $\tau_{\text {eff }}$ for samples of sufficient thickness. This is different for the limit of very small thickness, i.e. $d \rightarrow 0$. In this limit, the measurable lifetime vanishes even for a finite value of $\tau_{\mathrm{b}}$. This is caused by the strongly increased impact of the surface recombination on the overall measurement. Between these two limits there is a regime of intermediate thickness where a change in $\tau_{\mathrm{b}}$ is reflected in some change of $\tau_{\text {eff }}$. The onset of this regime can be estimated by a critical thickness $d_{\text {crit }}$ beyond which a measurement of the effective lifetime allows for a determination of the bulk lifetime. An analytic expression for this critical thickness in terms of the surface recombination $S$, the diffusion length $l$ (bulk lifetime $\tau_{\mathrm{b}}$ ) and absorption length $\alpha^{-1}$ is derived in the Sec. IV] Qualitatively, it is clear that 


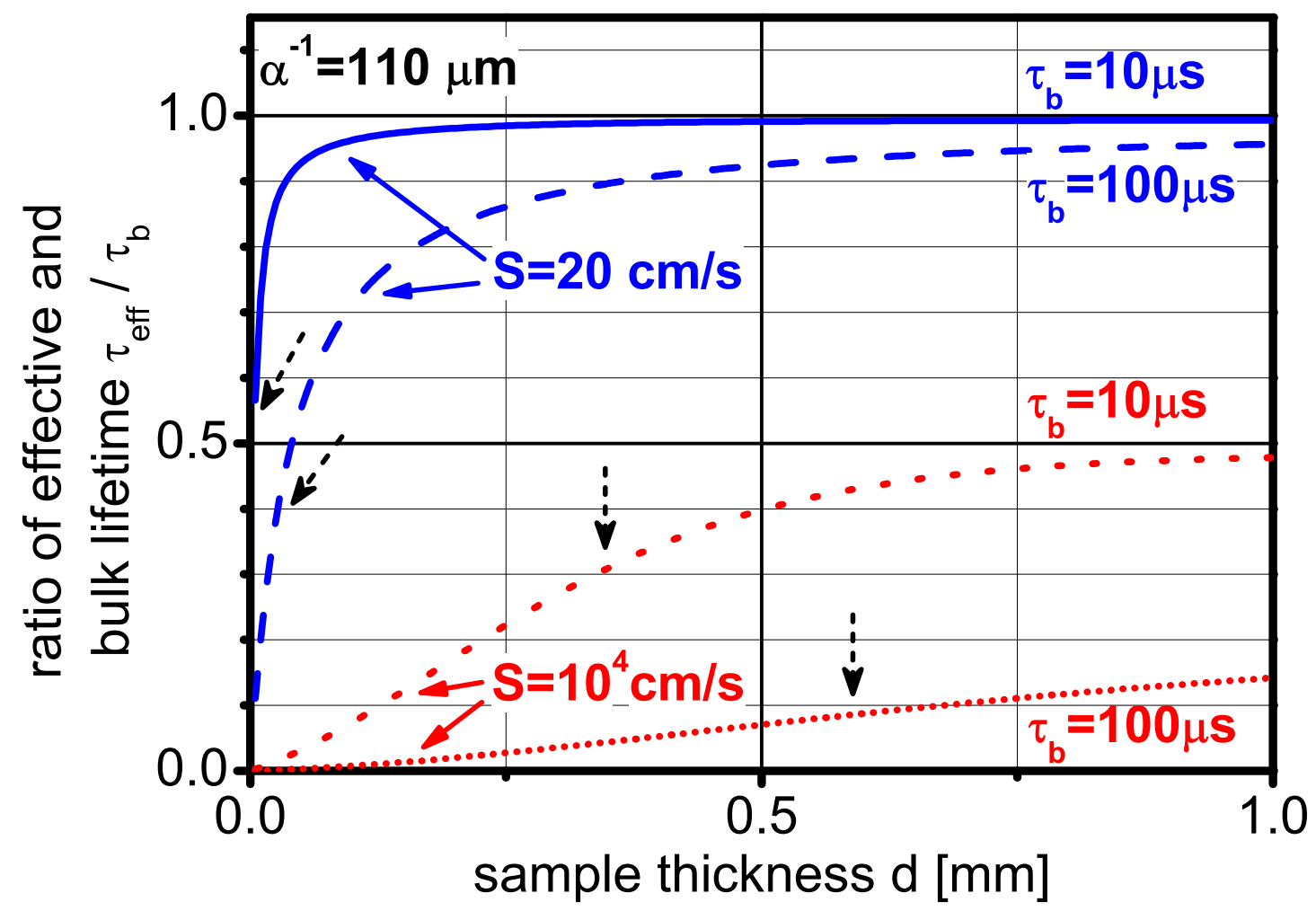

FIG. 3. (Color online) Ratio between measurable lifetime $\tau_{\text {eff }}$ and bulk lifetime $\tau_{\mathrm{b}}$ as a function of the sample thickness $d$. Shown are two different material qualitites: $\tau_{\mathrm{b}}=10 \mu \mathrm{s}$ (solid and dotted line) and $\tau_{\mathrm{b}}=100 \mu \mathrm{s}$ (dashed and short dashed line). The surface recombination velocity is chosen to be $S=20 \mathrm{~cm} / \mathrm{s}$ (upper curves, blue) and $S=10^{4} \mathrm{~cm} / \mathrm{s}$ (lower curves, red). The absorption length is $\alpha^{-1}=110 \mu \mathrm{m}$ for all four cases. The dashed arrows indicate the critical thickness $d_{\text {crit }}$, see Sec. IV,

a larger surface recombination velocity and a larger bulk lifetime lead to a larger critical thickness, see Fig. 3 .

\section{ASYMPTOTIC EXPRESSIONS}

Quantitative conclusions can be drawn by considering various limits of Eq. (5) with respect to the dimensionless surface recombination velocity $\bar{S}$, the absorption length $\bar{\alpha}^{-1}$, and the bulk lifetime $\bar{\tau}_{\mathrm{b}}=\bar{l}^{2}$. In terms of these parameters, Eq. (5) can be rewritten as

$$
\tau_{\mathrm{eff}}=\tau_{\mathrm{b}}\left[1-\mathcal{F}_{1}(\bar{S}, \bar{l}) \cdot \mathcal{F}_{2}(\bar{\alpha}, \bar{l})\right]
$$


with

$$
\mathcal{F}_{1} \equiv \frac{2 \bar{S} \bar{l}^{2}}{1+\bar{S} \bar{l} \operatorname{coth} \frac{1}{2 l}}, \quad \mathcal{F}_{2} \equiv \frac{\bar{\alpha}}{2} \frac{\operatorname{coth} \frac{\bar{\alpha}}{2}-\bar{\alpha} \bar{l} \operatorname{coth} \frac{1}{2 l}}{1-\bar{\alpha}^{2} \bar{l}^{2}}
$$

where $\mathcal{F}_{1}$ depends on $\bar{S}$ and $\bar{l}$ only while $\mathcal{F}_{2}$ is a function of $\bar{\alpha}$ and $\bar{l}$ only. Using the result (10) and the definition $\tau_{\mathrm{s}}=\left(\tau_{\text {eff }}^{-1}-\tau_{\mathrm{b}}^{-1}\right)^{-1}$ one finds

$$
\tau_{\mathrm{s}}=\tau_{\mathrm{s}}(\bar{l}, \bar{S}, \bar{\alpha})=\tau_{\mathrm{b}}\left[\mathcal{F}_{1}^{-1}(\bar{S}, \bar{l}) \mathcal{F}_{2}^{-1}(\bar{\alpha}, \bar{l})-1\right]
$$

The measurement $\tau_{\text {eff }}$ is hence dominated by the bulk property $\tau_{\mathrm{b}}$ if $\tau_{\mathrm{s}} / \tau_{\mathrm{b}} \gg 1$, i.e. for $\mathcal{F}_{1} \mathcal{F}_{2} \rightarrow 0$. The other limit of $\mathcal{F}_{1} \mathcal{F}_{2} \rightarrow 1$ implies $\tau_{\mathrm{s}} / \tau_{\mathrm{b}} \ll 1$ which means that the surface properties dominate and $\tau_{\text {eff }} \approx \tau_{\mathrm{s}}$.

The Eqs. (10) and (12) can be simplified for various applications by means of the approximation

$$
x \operatorname{coth} \frac{x}{2} \approx \begin{cases}x & \text { for } x \gg 3 \\ 2+x^{2} / 6-x^{4} / 360 & \text { for } x \ll 3\end{cases}
$$

together with standard series expansions. In the intermediate regime between the two limiting cases, i.e. at $x \approx 3$, this aproximation gives an error that is at most around $10 \%$. The application of the approximation to $\mathcal{F}_{1}$ and $\mathcal{F}_{2}$ leads, for example, in a direct way to the approximations of Eq. (10) that are applicable for small and large sample thickness $d$, i.e.

$$
\mathcal{F}_{1}(\bar{S}, \bar{l}) \approx \begin{cases}2 \bar{S} \bar{l}^{2} /\left(1+\bar{S}\left[2 \bar{l}^{2}+1 / 6\right]\right) & \text { for } \bar{l}>0.3 \\ 2 \bar{S} \bar{l}^{2} /(1+\bar{S} \bar{l}) & \text { for } \bar{l}<0.3\end{cases}
$$

and

$$
\mathcal{F}_{2}(\bar{\alpha}, \bar{l}) \approx \begin{cases}1+\bar{\alpha}^{2} /\left(720 \bar{l}^{2}\right) & \text { for } \bar{\alpha}<3, \bar{l}>0.3 \\ \bar{\alpha} /(2+2 \bar{\alpha} \bar{l}) & \text { for } \bar{\alpha}>3, \bar{l}<0.3\end{cases}
$$

respectively.

To illustrate the approach based on the two functions $\mathcal{F}_{1,2}$ and the applicability of the result Eq. (10), the following practical examples for p-type Silicon are considered (see Table 1 for details on the chosen parameters):

1. Surface-passivated wafer of low to intermediate material quality implying intermediate $\bar{l}$, small $\bar{S}$ and $\bar{\alpha}$ : In this limit one finds $\mathcal{F}_{1} \approx 2 \bar{S} \bar{l}^{2}$ and $\mathcal{F}_{2} \approx 1+\bar{\alpha}^{2} /\left(720 \bar{l}^{2}\right)$. Thus, according to the chosen parameters (see Table I), $\mathcal{F}_{1} \cdot \mathcal{F}_{2} \ll 1$ is a small correction in Eq. (10) which in this limit then reads $\tau_{\text {eff }} \approx \tau_{\mathrm{b}}\left[1-2 \bar{S} \bar{l}^{2}\right]$. This relation describes the curves shown in Fig. 2 for small $S$. Furthermore one can deduce the surface recombination time which is given 
by $\tau_{\mathrm{s}} \approx \tau_{\mathrm{b}} /\left(\mathcal{F}_{1} \mathcal{F}_{2}\right) \approx d /(2 S)$. Therefore, one obtains the same result as for the transient approach if the leading order in the small correction is considered.

2. Wafer of low to intermediate material quality without surface passivation characterized by intermediate $\bar{l}$, small $\bar{\alpha}$, and large $\bar{S}$ : In this case the asymptotic expressions are $\mathcal{F}_{1} \approx$ $2 \bar{S} \bar{l}^{2} /\left(1+2 \bar{S} \bar{l}^{2}+\bar{S} / 6\right)$ and $\mathcal{F}_{2} \approx 1+\bar{\alpha}^{2} /\left(720 \bar{l}^{2}\right)$. In contrast to case $1, \bar{S}$ is not small implying that $\mathcal{F}_{1} \cdot \mathcal{F}_{2}$ is not a small correction in Eq. (10) and therefore the measured $\tau_{\text {eff }}$ is much smaller than the bulk value $\tau_{\mathrm{b}}$. This is the behaviour presented in Fig. 2 for large $S$. Qualitatively, this is not surprising as the measured lifetime is governed by the large surface recombination. Practically, it allows for an estimation of an upper bound of the surface recombination velocity $S$ by assuming $\tau_{\mathrm{s}} \approx \tau_{\text {eff }}$. The surface recombination time $\tau_{\mathrm{s}}$ relates to $S$ via $\tau_{\mathrm{s}} \approx d /(2 S) \cdot[1+S d /(6 D)]$ which is found from Eq. (12). Since $S$ is not small, the second term in the brackets cannot be neglected as in the first case. The inverse relation $S \approx d /\left(2 \tau_{\mathrm{s}}\right)\left[1-d^{2} /\left(12 D \tau_{\mathrm{s}}\right)\right]^{-1}$ can then be employed to calculate $S$.

3. Surface-passivated float-zone wafer (very high material quality) with large $\bar{l}$, small $\bar{S}$, and small $\bar{\alpha}$ : The asymptotic behaviour of $\mathcal{F}_{1}$ and $\mathcal{F}_{2}$ is analogous to the previous case, i.e. $\mathcal{F}_{1} \approx 2 \bar{S} \bar{l}^{2} /\left(1+2 \bar{S} \bar{l}^{2}+\bar{S} / 6\right)$ and $\mathcal{F}_{2} \approx 1+\bar{\alpha}^{2} /\left(720 \bar{l}^{2}\right)$, leading to the same expressions for $\tau_{\text {eff }}$ and $\tau_{\mathrm{s}}$ when inserted in Eqs. (10) and (12). Again, the measurement of $\tau_{\text {eff }}$ is dominated by surface effects allowing for the estimation of an upper bound of the surface recombination velocity $S$.

4. Block of low to intermediate material quality without surface passivation describable by small $\bar{l}$, large $\bar{S}$ and $\bar{\alpha}$ : The asymptotics in this case read $\mathcal{F}_{1} \approx 2 \bar{S} \bar{l}^{2} /(1+\bar{S} \bar{l})$ and $\mathcal{F}_{2} \approx \bar{\alpha} /(2+2 \bar{\alpha} \bar{l})$. Together with Eq. (10) this exactly reproduces the result $\tau_{\text {eff }}^{d \rightarrow \infty}$. Again, the product $\mathcal{F}_{1} \cdot \mathcal{F}_{2}$ is not a small correction. Nevertheless, the bulk information can be extracted from the measurement.

It is clear from the previous discussion and from Fig. 1 that a measurement of a sample of lower material quality is more likely to give an accurate estimate of the bulk property $\tau_{\mathrm{b}}$. This is reflected by the fact that $\tau_{\text {eff }} / \tau_{\mathrm{b}} \rightarrow 1$ for $\tau_{\mathrm{b}} \rightarrow 0$. However, in most pratical cases one cannot expect the relation $\tau_{\text {eff }}=\tau_{\mathrm{b}}$ to be exact and the corrections to this equality are 


$\left.\begin{array}{|c||c|c|c||c|c|c||c|c|}\hline \text { case } & \begin{array}{c}\tau_{\mathrm{b}} \\ {[\mu \mathrm{s}]}\end{array} & \begin{array}{c}d \\ {[\mathrm{~mm}]}\end{array} & S & \bar{l}=\frac{l}{d} & \bar{S}=\frac{S d}{D} & \bar{\alpha}=\alpha d & \begin{array}{c}\tau_{\mathrm{eff}} \\ {[\mathrm{cm} / \mathrm{s}]}\end{array} & \begin{array}{c}\tau_{\mathrm{s}} \\ {[\mu \mathrm{s}]}\end{array} \\ {[\mu \mathrm{s}]}\end{array}\right]$

TABLE I. Dimensionless parameters $\bar{l}, \bar{S}$, and $\bar{\alpha}$ for the examples under consideration. The absorption length has been chosen to be constant for all cases, i.e. $\alpha^{-1}=110 \mu \mathrm{m}$ ), while the diffusion constant is set to $D=30 \mathrm{~cm}^{2} / \mathrm{s}$.

of interest. Using the asymptotics of $\mathcal{F}_{1} \approx 2 \bar{S} \bar{l}^{2}$ and $\mathcal{F}_{2} \approx \bar{\alpha}[\operatorname{coth}(\bar{\alpha} / 2)-\bar{\alpha} \bar{l}] / 2$ yields

$$
\begin{aligned}
\tau_{\mathrm{eff}}^{\tau_{\mathrm{b}} \rightarrow 0} & =\tau_{\mathrm{b}}\left[1-\bar{l}^{2} \bar{S} \bar{\alpha} \operatorname{coth} \frac{\bar{\alpha}}{2}\right] \\
& \approx\left\{\begin{array}{l}
\tau_{\mathrm{b}}\left[1-2 \bar{S} \bar{l}^{2}\right] \text { for } \bar{\alpha} \ll 1 \\
\tau_{\mathrm{b}}\left[1-\bar{\alpha} \bar{S} \bar{l}^{2}\right] \text { for } \bar{\alpha} \gg 1
\end{array}\right.
\end{aligned}
$$

indicating the first order correction in $\bar{\tau}_{\mathrm{b}} \equiv \bar{l}^{2}$ being valid for material of poor quality (low bulk lifetimes). The criterion for how large the bulk lifetime of a sample of fixed thickness and surface quality can become before a measurement of $\tau_{\text {eff }}$ does not relate to bulk properties anymore is hence given by the correction being much smaller than one. There is a rather intuitive interpretation for this. For homogeneous generation, i.e. $\bar{\alpha} \ll 1$, the condition $2 \bar{S} \bar{l}^{2}=2 S \tau_{\mathrm{b}} / d=\tau_{\mathrm{b}} / \tau_{\mathrm{s}} \ll 1$ just means that the two regions where the surface recombination dominates are of thickness $2 S \tau_{\mathrm{b}}$ and have to be negligible compared to the total sample thickness $d$. For strongly inhomogeneous generation, i.e. $\bar{\alpha} \gg 1$, the criterion is replaced by $\bar{\alpha} \bar{S} \bar{l}^{2}=S \tau_{\mathrm{b}} \alpha \ll 1$ meaning that the width $S \tau_{\mathrm{b}}$ of the one region with dominating surface recombination has to be smaller than the absorption depth $\alpha^{-1}$.

On the other hand, the measured lifetime of a sample of very high material quality, i.e. very large bulk lifetime, is mostly dominated by the surface effects. In particular, this means that $\tau_{\text {eff }}^{\tau_{\mathrm{b}} \rightarrow \infty}$ saturates at a value that is independent of $\tau_{\mathrm{b}}$, i.e. $\tau_{\text {eff }}^{\tau_{\mathrm{b}} \rightarrow \infty} \rightarrow \tau_{\mathrm{s}}$. With the appropriate asymptotic expressions for $\mathcal{F}_{1}$ and $\mathcal{F}_{2}$ one finds

$$
\tau_{\mathrm{eff}}^{\tau_{\mathrm{b}} \rightarrow \infty}=\tau_{\mathrm{s}}^{\tau_{\mathrm{b}} \rightarrow \infty}=t_{\mathrm{cr}} \frac{\bar{\alpha}^{2}-2 \bar{S}+\bar{S} \bar{\alpha} \operatorname{coth} \frac{\bar{\alpha}}{2}}{2 \bar{S} \bar{\alpha}^{2}}
$$

In other words, a measurement on a sample of very high material quality gives information 
on the quality of the surface passivation, i.e. on $\tau_{\mathrm{s}}$, rather than on $\tau_{\mathrm{b}}$. The limit (14) can thus be used to determine $S$ for a given thickness $d$ and absorption length $\alpha^{-1}$. This limit together with the general solution (10) suggests the large $\bar{l} \gg 1$ structure $\tau_{\text {eff }}^{\bar{l} \gg 1}=$ $\tau_{\mathrm{b}}\left[1-A^{*} 2 \bar{l} \tanh (1 /\{2 \bar{l}\})\right]$ with $A^{*}=1-\tau_{\text {eff }}^{\tau_{\mathrm{b}} \rightarrow \infty} /\left(t_{\mathrm{cr}} \bar{l}^{2}\right)$ which is slightly different from the phenomenological proposal for unpassivated wafers in Ref. 16 with respect to the prefactor $A^{*}$. In the special case of thin samples with homogeneous generation, i.e. wafers with $\bar{\alpha} \rightarrow 0$, the limit (14) reduces to

$$
\tau_{\mathrm{eff}}^{\tau_{\mathrm{b}} \rightarrow \infty, \alpha \rightarrow 0}=\tau_{\mathrm{s}}^{\tau_{\mathrm{b}} \rightarrow \infty, \alpha \rightarrow 0}=t_{\mathrm{cr}} \frac{6+\bar{S}}{12 \bar{S}}=\frac{d}{2 S}+\frac{d^{2}}{12 D} .
$$

Note, that this result for the steady state approach is similar but not equal to the surface recombination approximation $\tau_{\mathrm{s}, \text { tr }}$ obtained in the transient case. The difference lies in the numerical prefactor of the term $\sim d^{2}$ that is relevant for samples of larger thickness.

Experimentally, an adjustment of the bulk lifetime is typically more difficult than the preparation of a sample with a specified thickness. Therefore, the approximate behaviour in the limit of small and large sample thickness is of particular interest. The ratio $\tau_{\text {eff }} / \tau_{\mathrm{b}}$ saturates at a constant value smaller than one for very thick samples, see Fig. 3. In particular, one finds with the asymptotics $\mathcal{F}_{1} \approx 2 \bar{S} \bar{l}^{2} /(1+\bar{S} \bar{l})$ and $\mathcal{F}_{2} \approx \bar{\alpha} /(2+2 \bar{\alpha} \bar{l})$ the result

$$
\frac{\tau_{\mathrm{eff}}^{d \rightarrow \infty}}{\tau_{\mathrm{b}}} \approx 1-\frac{\bar{S} \bar{\alpha} \bar{l}^{2}}{(1+\bar{S} \bar{l})(1+\bar{\alpha} \bar{l})}=1-\frac{\alpha l}{1+\alpha l} \frac{S l}{D+S l}
$$

which is exactly the result presented in Sec. III for blocks. This relationship allows for a determination of $\tau_{\mathrm{b}}$ as a function of $\tau_{\text {eff }}$. In general, the knowledge of $S$ would be required in order to apply this relation. However, it becomes clear from Eq. (16) that for unpassivated blocks with large $S$ the influence of this parameter is rather weak and can thus be neglected. On the other hand, the ratio $\tau_{\text {eff }} / \tau_{\mathrm{b}}$ vanishes for small thicknesses which is caused by the surface effects. The asymptotic behaviour for $d \rightarrow 0$ is obtained from $\bar{\alpha}, \bar{S} \ll 1$ and $\bar{l} \gg 1$ giving $\mathcal{F}_{1} \approx 2 \bar{S} \bar{l}^{2} /\left[1+\bar{S}\left(2 \bar{l}^{2}+1 / 6\right)\right]$ and $\mathcal{F}_{2} \approx 1+\bar{\alpha}^{2} /\left(720 \bar{l}^{2}\right)$. Quantitatively, one thus finds

$$
\frac{\tau_{\mathrm{eff}}^{d \rightarrow 0}(d)}{\tau_{\mathrm{b}}} \approx 1-\frac{2 \bar{S} \bar{l}^{2}}{1+2 \bar{S} \bar{l}^{2}+\bar{S} / 6}=1-\frac{2 S l^{2}}{D d+2 S l^{2}+S d^{2} / 6}
$$

which is correct up to the second order in $d$. Hence, a determination of $\tau_{\mathrm{b}}$ from $\tau_{\text {eff }}$ is not possible in this regime since $\tau_{\text {eff }} \approx d /(2 S)$ for $d \rightarrow 0$ depends only on $S$ but not on $\tau_{\mathrm{b}}$. Therefore, the lifetime measurement on thin wafers can be used to determine $S$ but it does not allow to deduce the bulk property $\tau_{\mathrm{b}}$. The critical thickness that separates the 
two regimes of large thickness, Eq. (16), and small thickness, Eq. (17) can be estimated by $\tau_{\text {eff }}^{d \rightarrow \infty} / 2=\tau_{\text {eff }}^{d \rightarrow 0}\left(d_{\text {crit }}\right)$, see Fig. 3. Using Eq. (16) and Eq. (17) the corresponding quadratic equation in $d_{\text {crit }}$ reads

$$
d_{\text {crit }}^{2}+\frac{6 D}{S} d_{\text {crit }}+12 l^{2} \frac{\alpha l^{2} S-(1+\alpha l)(D+S l)}{(1+\alpha l)(D+S l)+\alpha l^{2} S}=0
$$

which can easily be solved. The two most interesting cases are given by small $S$ (passivated samples) and large $S$ (unpassivated samples) for which the solutions are

$$
d_{\mathrm{crit}}^{S \rightarrow 0} \approx 2 \tau_{\mathrm{b}} S \text { and } d_{\mathrm{crit}}^{S \rightarrow \infty} \approx \sqrt{6 \frac{l}{\alpha}}
$$

respectively. There is an intuitive interpretation of these results. For samples with a good surface passivation a measurement of the bulk properties is possible if the sample is more than twice the width $\tau_{\mathrm{b}} S$ of the regions where the surface recombination takes place. This picture cannot be applied in the case of low surface passivation with large $S$ since the nearsurface regions of width $\tau_{\mathrm{b}} S$ would extend throughout the entire sample. In this case, other length scales are important, in particular the diffusion length $l$ and the absorption length $\alpha^{-1}$. The critical thickness is then simply the geometric mean of these two length scales.

\section{CONCLUSIONS}

In this work, the generalized solution for the relation between the measured lifetime and the bulk lifetime of excess minority carriers is investigated. This relation allows to study the interplay of the bulk and surface recombination for a steady-state measurement. It provides a general theoretical background for a consistent data analysis of lifetime measurements on a wide variety of samples. In particular, it allows identifying the influence of the relevant parameters, i.e. bulk lifetime $\tau_{\mathrm{b}}$, surface recombination velocity $S$, absorption depth $\alpha^{-1}$, and sample thickness $d$ on the measured lifetime $\tau_{\text {eff }}$. The practically most important cases of passivated and unpassivated wafers and blocks are discussed within the same framework and exact analytic relations for these cases are presented. The proposed approach is demonstrated for typical and practical relevant examples. It is shown that a reliable measurement of the bulk properties for given surface conditions is possible only if the sample is thick enough and the surface passivation sufficiently effective. On the other hand, lifetime measurements on samples thinner than a critical thickness $d_{\text {crit }}$ are best employed when the 
quality of the surface passivation layer is of interest. For an unambiguous determination of both $\tau_{\mathrm{b}}$ and $S$ at least two samples of intermediate thickness have to be measured. Alternatively, an experimental setup where only one sample is analyzed with a light source of two different wave lengths, i.e. two different $\alpha$, can give this information as well. These qualitative conclusions are substantiated by the corresponding quantitative relations which allow an accurate analysis of experimental data. Among those, an estimate of the relevant critical thickness in dependence on the passivation, i.e. value of $S$, and illumination, i.e. value of $\alpha^{-1}$, is derived.

\section{ACKNOWLEDGMENTS}

The author acknowledges the funding of the Ministerium für Wirtschaft und Arbeit des Landes Sachsen-Anhalt within the ESCA Project (Project FuE 62/09).

\section{REFERENCES}

${ }^{1}$ D. K. Schroder, Semiconductor material and device characterization (John Wiley And Sons, Inc., Hoboken, New Jersey, 2006).

${ }^{2}$ S. Rein, Lifetime Spectroscopy (Springer-Verlag, 2005).

${ }^{3}$ K. Luke and L.-J. Cheng, J. Appl. Phys. 61, 2282 (1987).

${ }^{4}$ V. Grivickas, D. Noreika, and J. Tellesfen, Lithuanian Physics Journal 29, 591 (1989).

${ }^{5}$ G. Kousik, Z. Ling, and P. Ajmera, J. Appl. Phys. 72, 141 (1992).

${ }^{6}$ T. Otaredian, Solid-State Electronics 36, 153 (1993).

${ }^{7}$ A. Sproul, J. Appl. Phys. 76, 2851 (1994).

${ }^{8}$ H. B. DeVore, Phys. Rev. 102, 86 (1956).

${ }^{9}$ G. Duggan and G. B. Scott, J. Appl. Phys. 52, 407 (1980).

${ }^{10}$ H. Nagel, C. Berge, and A. G. Aberle, J. Appl. Phys. 86, 6218 (1999).

${ }^{11}$ J. Brody, A. Rohatgi, and A. Ristow, Solar Energy Materials and Solar Cells 77, 293 (2003).

${ }^{12}$ R. Sinton and A. Cuevas, Applied Physics Letters 69, 2510 (1996).

${ }^{13}$ A. G. Aberle, Progress in Photovoltaics: Research and Applications 8, 473 (2000).

${ }^{14}$ J. Schmidt and A. G. Aberle, J. Appl. Phys. 81, 6186 (1997),

${ }^{15}$ S. Bowden and R. A. Sinton, J. Appl. Phys. 102 (2007), 10.1063/1.2818371. 
${ }^{16}$ R. Sinton, H. Tathgar, S. Bowden, and A. Cuevas, in Proceedings of the 14th NREL Workshop on Crystalline Silicon Solar Cell Materials and Processes (2004).

${ }^{17}$ K. Bothe, R. Krain, R. Falster, and R. Sinton, Prog. Photovolt.: Res. Appl. 18, 204 (2010).

${ }^{18}$ J. Brody and A. Rohatgi, Solid-State Electronics 46, 859 (2002).

${ }^{19}$ M. Bail and R. Brendel, in 16th European Photovoltaic Solar Energy Conference (2000). 